\title{
PANAYIOTOPOULOS SYNDROME: BENIGN SYSTEM EPILEPSY
}

The clinical and EEG features of Panayiotopoulos syndrome (PS) are reviewed and the pathophysiology discussed in a report from Guy's, St Thomas" and Evelina Hospital for Children, London, UK. Autonomic seizures are the clinical hallmark of PS. Vomiting occurs in $70-80 \%$ of seizures. Other autonomic symptoms and signs (AS/S) include mydriasis, cardiorespiratory, gastrointestinal and thermoregulatory alterations, incontinence, coughing, and hypersalivation. Rarely, cardiorespiratory arrest may occur. Awareness is intact initially, but is usually impaired later. Ictal syncope is used to describe a seizure in which the child is unresponsive, pale with blue lips, and flaccid. Seizures may be purely autonomic or combined with other ictal features. About $10 \%$ patients have exclusively nonautonomic seizures. Half of the seizures last from 30 min to hours, sometimes becoming an autonomic status epilepticus. They occur during nocturnal sleep or brief daytime naps.

Typical EEG findings include focal or multifocal interictal spikes, worse during sleep, and mainly occipital. In some, the spikes are centrotemporal/parietal, similar to benign rolandic epilepsy. A small number of ictal recordings document occipital and extraoccipital onsets.

Spontaneous remission is the rule, within 2 years from onset. Antiepileptic treatment should be reserved for those children with frequent or prolonged seizures. Carbamazepine is the agent usually preferred, although one case of seizure exacerbation is recorded, and caution is required. PS is generally benign, but some seizures are prolonged and very rarely, may be attended by cardiorespiratory arrest.

An attempt to explain the pathophysiology of PS distinguishes between idiopathic autonomic and symptomatic focal seizures with AS/S. The ILAE recent task force classifies PS as "early onset benign childhood occipital epilepsy-Panayiotopoulos type." PS may be considered an example of "system" epilepsy, an alternative term to "idiopathic." PS represents an idiopathic focal epilepsy, with different characteristics from symptomatic focal epilepsy with AS/S in which vomiting is infrequent, usually occurring postictally. (Koutroumanidis M. Panayiotopoulos syndrome: an important electroclinical example of benign childhood system epilepsy. Epilepsia July 2007;48:1044-1053). (Reprints: Dr Michael Koutroumanidis, Department of Clinical Neurophysiology and Epilepsies, Guy's St Thomas' Hospital and Evelina Hospital for Children NHS Trust, Lambeth Wing, $3^{\text {rd }}$ floor, London, UK).

COMMENT. PS is characterized by prolonged seizures with prominent autonomic features and multifocal EEG spikes occurring in developmentally normal children.

A prospective study of $\mathbf{1 9 2}$ patients with PS in Buenos Aries, Argentina, included sleep and awake EEGs obtained between Feb 1990 and 2006. (Caraballo R, Cersosimo R, Fejerman N. Epilepsia July 2007;48:1054-1061). In the same period, 398 children with BCECTS were identified. PS had a peak age at onset of 5 years. Autonomic manifestations were most frequent with ictal vomiting occurring in $160(82.3 \%)$ children. Eye and head deviation occurred in $170(88 \%)$, clonic partial seizures in $30.7 \%$, visual symptoms in $10.4 \%$, and secondary generalized seizures in $36.4 \%$. One third had partial status epilepticus. EEG showed occipital spikes in $75 \%$. Family history of epilepsy was obtained in $31.3 \%$, including 4 pairs of siblings with PS. Febrile seizures occurred in $28.5 \%$ of patients and were reported in $13.5 \%$ of family members. 